\title{
Convergent and Asymptotic Iteration Methods in General Relativity
}

\author{
D. Christodoulou and B. G. Schmidt \\ Max-Planck-Institut für Physik und Astrophysik, D-8000 München 40, FRG
}

\begin{abstract}
We show that the fast motion iteration method in General Relativity gives an asymptotic approximation to exact solutions of the reduced Einstein equations. Rigorous estimates of the error commited at each step of the iteration are derived.
\end{abstract}

\section{Introduction}

In General Relativity powerful theorems are known, which guarantee existence and uniqueness of solutions to Einstein's equations [1-4] under very general conditions. In contrast to this, however, very little is known about the validity of the approximation methods, on which the comparison between theory and observations is based. One has in fact reasons to be rather sceptical about the usual procedures.

The main result of this paper is an exact estimate of the error one makes by solving the reduced Einstein equations in the usual fast motion iteration scheme.

We outline the basic arguments with the following model problem: Solve, for a given source $\varrho(x, y, z, t)$, and for a finite time interval $0 \leqq t \leqq T$, the quasilinear equation

$$
\square_{\phi} \phi:=\left(\square+\phi \partial_{x}^{2}\right) \phi=\varrho, \quad \square=-\partial_{t}^{2}+\partial_{x}^{2}+\hat{o}_{y}^{2}+\partial_{z}^{2}
$$

with zero initial data, i.e., $\phi=\partial_{t} \phi=0$ for $t=0$, in which case all properties of the solution should be determined by the source. In the scetch, which we shall give in this introduction, \|\| will stand for norms in Banach spaces, which will be specified later in the text. Further " $c$ " will stand for a constant depending only on $T$.

The existence theorems for the problem (1.1) are based on the iteration

$$
\square_{\phi_{n}} \phi_{n+1}=\varrho, \quad \phi_{0}=0 .
$$

If $\varrho$ is small enough this sequence has the property

$$
\left\|\phi_{n+1}-\phi_{n}\right\| \leqq \lambda\left\|\phi_{n}-\phi_{n-1}\right\|, \quad 0<\lambda<1,
$$


which shows that $\phi_{n}$ is a Cauchy sequence in a Banach space, hence it converges to a unique solution $\phi$. Furthermore

$$
\left\|\phi-\phi_{n}\right\| \leqq \frac{\lambda^{n}}{1-\lambda}\left\|\phi_{1}-\phi_{0}\right\|=\frac{\lambda^{n}}{1-\lambda}\left\|\phi_{1}\right\| \text {. }
$$

The contraction constant $\lambda$ as well as $\left\|\phi_{1}\right\|$ can both be bounded by a norm of the source $\varrho$, hence

$$
\left\|\phi-\phi_{n}\right\| \leqq c\|Q\|^{n+1} \text {. }
$$

Suppose we want to actually calculate the iterates $\phi_{n}$. Then we face the problem of constructing the Greens functions of $\square_{\phi_{n}}$, which is not possible analytically. The usual way around this difficulty is to rewrite (1.1) as

$$
\square \phi=\varrho-\phi \partial_{x}^{2} \phi
$$

and to iterate as follows:

$$
\square \bar{\phi}_{n+1}=\varrho-\bar{\phi}_{n} \partial_{x}^{2} \bar{\phi}_{n}, \quad \bar{\phi}_{0}=0 \text {. }
$$

Here we have to solve in each step just a flat space wave equation, whose source is determined by $\varrho$ and the $\bar{\phi}_{n}$, calculated before. In general, however, this sequence $\left(\bar{\phi}_{n}\right)$ can not be expected to converge, as can be seen from domain of dependence arguments.

Unfortunately, we have now two equally useless sequences: $\left\{\phi_{n}\right\}$ converges, but cannot be calculated, whereas $\left\{\bar{\phi}_{n}\right\}$ can be calculated, but we don't know whether and how the $\bar{\phi}_{n}$ 's are related to the exact solution $\phi$.

In Einstein's theory of gravitation exactly the same difficulty occurs.

Nevertheless, it is possible to show, as we shall now outline, that the $\left\{\bar{\phi}_{n}\right\}$ sequence is in fact asymptotic to the exact solution $\phi$ as $\varrho \rightarrow 0$ (in a sense to be specified in the text) and the error commited at each step can be estimated.

Combine the two iterations (1.5) and (1.7). One gets by eliminating $\varrho$

$$
\square_{\bar{\phi}_{n}}\left(\phi_{n+1}-\bar{\phi}_{n+1}\right)=\left(\phi_{n}-\bar{\phi}_{n}\right) \partial_{x}^{2} \phi_{n+1}+\bar{\phi}_{n} \partial_{x}^{2}\left(\bar{\phi}_{n+1}-\bar{\phi}_{n}\right)
$$

provided one subtracts $\square_{\bar{\phi}_{n}} \phi_{n+1}$ on both sides. This is a linear wave equation for $\phi_{n+1}-\bar{\phi}_{n+1}$ with wave operator $\square \bar{\phi}_{n}$ and a source depending only on quantities of the $\bar{\phi}_{n}$ iteration, $\partial_{x}^{2} \phi_{n+1}$ and the difference $\left(\phi_{n}-\bar{\phi}_{n}\right)$. We have in this case an energy inequality which boundes the unknown $\phi_{n+1}-\bar{\phi}_{n+1}$ in terms of the source. Hence one can estimate (provided that we can brake up the norm of the products into the product of the norms which we shall show in the text)

$$
\left\|\phi_{n+1}-\bar{\phi}_{n+1}\right\| \leqq c\left\{\left\|\phi_{n}-\bar{\phi}_{n}\right\|\left\|\partial_{x}^{2} \phi_{n+1}\right\|+\left\|\bar{\phi}_{n}\right\|\left\|\partial_{x}^{2}\left(\bar{\phi}_{n+1}-\bar{\phi}_{n}\right)\right\|\right\} .
$$

Since all three terms $\left\|\partial_{x}^{2} \phi_{n+1}\right\|,\left\|\bar{\phi}_{n}\right\|$ and $\left\|\partial_{x}^{2}\left(\bar{\phi}_{n+1}-\bar{\phi}_{n}\right)\right\|$ can be estimated by $\|\varrho\|$ one finds a recursion relation of the form:

$$
\left\|\phi_{n+1}-\bar{\phi}_{n+1}\right\| \leqq c\|\varrho\|\left\|\phi_{n}-\bar{\phi}_{n}\right\|+c\|\varrho\|^{n+2}
$$

which can easily be solved (since the first member $\phi_{1}-\bar{\phi}_{1}=0$ ), provided the norms of $\left(\phi_{n+1}-\bar{\phi}_{n+1}\right)$ and $\left(\phi_{n}-\bar{\phi}_{n}\right)$ are taken in the same Banach space (a fact which we shall show in the text). Thus

$$
\left\|\phi_{n}-\bar{\phi}_{n}\right\| \leqq c\|\varrho\|^{n+1} \text {. }
$$

Combining this with (1.5) the result is

$$
\left\|\phi-\bar{\phi}_{n}\right\| \leqq c\|Q\|^{n+1} \text {. }
$$


It will turn out that the norm of $\varrho$ will contain derivatives of $\varrho$ up to order $n+1$, i.e. the right hand side, in contrast to (1.5), is not in fact proportional to a power of a fixed number. Equation (1.12) shows that the sequence $\left\{\bar{\phi}_{n}\right\}$ is in fact asymptotic to the exact solution $\phi$.

Essentially the same estimate will be true for the reduced Einstein equations, i.e., Einstein's equation in a harmonic coordinate system

$$
g^{\alpha \beta} \partial_{\alpha} \partial_{\beta} g^{\mu v}+P^{\mu v}\left(g^{\kappa \lambda}, \partial_{\varrho} g^{\kappa \lambda}\right)=Q^{\mu \nu} .
$$

The difference between the exact solution $g^{\mu \nu}$ and an approximate solution $\bar{g}_{n}^{\mu \nu}$, calculated by an iteration analogous to (1.7) will be bounded by a power of a norm of the source,

$$
\left\|g-\bar{g}_{n}\right\| \leqq c\|Q\|^{n+1} .
$$

The method by which we estimate the difference of the true solution and the iterates of a non-converging iteration procedure can be applied to other problems of classical field theories.

The plan of the paper is the following:

In Sect. 2 the tools from functional analysis is outlined.

The analysis of the convergent and asymptotic iterations is given in Sects. 3 and 4 respectively, and their comparison and final theorem appears in Sect. 5. Concluding remarks and comments follow in Sect. 6.

\section{Function Spaces and Multiplication Properties}

We define the following function spaces on $I \times \mathbb{R}^{n}$, where $I$ is the interval $[0, T]$ [9].

Definition 1. $\tilde{E}_{s}$ is the space of tensor fields $h$ on $I \times \mathbb{R}^{n}$ such that

i) the restriction $h_{t}$ of $h$ as well as the restriction $\left(D^{\alpha} h\right)_{t}$ of its derivatives of any order $|\alpha| \leqq s$, to each $\Sigma_{t}=\mathbb{R}^{n} \times\{t\}$ is almost everywhere defined and square integrable.

We set

$$
\|h\|_{s}^{\Sigma_{t}}=\left[\int_{\Sigma_{t}} \sum_{|\alpha| \leqq s}\left|\left(D^{\alpha} h\right)_{t}\right|^{2} d x\right]^{1 / 2} \text {. }
$$

ii) The mapping $I \rightarrow \mathbb{R}$ by $t \rightarrow\|h\|_{s}^{\Sigma_{t}}$ is measurable and essentially bounded.

$\tilde{E}_{s}$ endowed with the norm:

$$
\|h\|_{s}=\text { Ess } \operatorname{Sup}_{t \in I}\|h\|_{s}^{\Sigma_{t}}
$$

is a Banach space.

We will need the following multiplication properties.

Lemma 1. If $k, l \geqq m$ and $k+l>m+\frac{n}{2}$ we have the continuous multiplication property

$$
\tilde{E}_{k} \times \tilde{E}_{l} \rightarrow \tilde{E}_{m}
$$


by

$$
(u, v) \rightarrow u \otimes v
$$

i.e., there are constants $a_{k, l, m}$ such that

$$
\|u v\|_{m} \leqq a_{k, l, m}\|u\|_{k} \cdot\|v\|_{l}
$$

Proof. On each $\Sigma_{t}$ we can show that

$$
\|u v\|_{m}^{\Sigma_{t}} \leqq a_{k, l, m}\|u\|_{k}^{\Sigma_{t}} \cdot\|v\|_{l}^{\Sigma_{t}}
$$

in the same manner in which the analogous inequality is established for the corresponding Sobolev spaces over $\Sigma_{t}[7,8]$. Taking the essential supremum in $I$ we get (2.5).

Corollary 1. If $k>\frac{n}{2}, \tilde{E}_{k}$ is a Banach algebra.

We shall write $a_{k, l, l}$ as $a_{k, l}$ and $a_{k, k}$ simply as $a_{k}$.

Lemma 2. If $s>\frac{n}{2}, \tilde{E}_{s}$ is continuously imbedded in $L^{\infty}\left(I, C_{b}^{0}\left(\mathbb{R}^{n}\right)\right)$ that is there exists a universal constant $c$ such that $\forall f \in \tilde{E}_{s}$

$$
\text { Ess } \operatorname{Sup}_{t \in I}\left\{|f|_{C_{b}}^{\Sigma_{t}}\right\} \leqq c\|f\|_{s} .
$$

Lemma 3. If the restriction $(f)_{t_{0}} \in L^{2}\left(\Sigma_{t_{0}}\right)$ and $D f \in \tilde{E}_{0}$ then $f \in \tilde{E}_{1}$ and

$$
\|f\|_{1}^{2} \leqq 2\left(|f|_{L^{2}}^{\Sigma_{t_{0}}}\right)^{2}+2 T^{2} \cdot\left(\|D f\|_{0}\right)^{2} .
$$

Definition 2. $\Sigma_{t}$ is uniformly space like with uniform lapse for a $C^{0}$ 2-contra-variant symmetric tensor field $\gamma$ on $I \times \mathbb{R}^{3}$ if there exist strictly positive constant $a_{0}, A_{0}, a_{1}$, $A_{1}, B$ such that on $\Sigma_{t}$

$$
\begin{aligned}
a_{0} & \leqq-\gamma^{00} \leqq A_{0} \\
a_{1}|\xi|^{2} & \leqq \gamma^{i k} \xi_{i} \xi_{k} \leqq A_{1}|\xi|^{2} \\
\left|\gamma^{0 i}\right| & \leqq B,
\end{aligned}
$$

where $\gamma^{\mu \nu}$ are the components of $\gamma$ in the natural frame on $I \times \mathbb{R}^{3}$.

Remark. The above inequalities imply the analogous inequalities for $\gamma_{\mu \nu}$. The three conditions are equivalent to the following geometrical restrictions:

1. The normals to $\Sigma_{t}$ lie always in a cone around $\frac{\partial}{\partial t}$.

2. The opening of the light cone of the metric $\gamma$ is bounded away from zero and infinity.

3. The lapse function on $\Sigma_{t}$ is bounded and greater than a positive constant. Definition 3. We say that a $C^{0}$ 2-contra-variant tensorfield $\gamma$ is a regularly hyperbolic metric on $I \times \mathbb{R}^{3}$ if $\Sigma_{t}$ is uniformly spacelike with uniform lapse for each $t \in I$ and if the constants $a_{0}, A_{0}, a_{1}, A_{1}$, and $B$ can be chosen independently of $t$. 
Let $\square_{\gamma}$ be the operator $\square_{\gamma}=\gamma^{\mu v} D_{\mu} D_{v}$ and consider the equation on $I \times \mathbb{R}^{3}$

$$
\square_{\gamma} \phi=\varrho
$$

with $\varrho$ given, for Cauchy data $\phi=\partial_{t} \phi=0$ on $\Sigma_{0}$. Denote by $\eta$ the Minkowski metric on $I \times \mathbb{R}^{3}$, which is diagonal $(-1,+1,+1,+1)$ in the natural coordinates and let $\chi$ be the difference $\chi=\gamma-\eta$. The following theorem was established in [9].

\section{Theorem 1}

Suppose: $1 . \gamma$ is a regularly hyperbolic metric on $I \times \mathbb{R}^{3}$.

2. $D \gamma \in L^{\infty}\left(I \times \mathbb{R}^{3}\right)$

3. $\chi \in \tilde{E}_{k}$

4. $\varrho \in \tilde{E}_{k-1}$

then there exists a solution $\phi$ in $\tilde{E}_{k}$ to the above Cauchy problem, unique in $\tilde{E}_{1}$, satisfying the inequality:

$$
\|\phi\|_{k} \leqq c_{k}^{\prime}\|\varrho\|_{k-1},
$$

where constants $c_{k}^{\prime}$ depend only on $a_{0}, A_{0}, a_{1}, A_{1}, B,|D \gamma|_{L^{\infty}},\|\chi\|_{k}$ and $T$.

Remark. If $\chi \in \tilde{E}_{k}$ with $k \geqq 3$, Lemma 2 implies that condition 2 is satisfied and that

$$
|\chi| \leqq c\|\chi\|_{k}
$$

everywhere on $I \times \mathbb{R}^{3}$. On the other hand it is easy to check that $\gamma$ is regularly hyperbolic if $|\chi| \leqq$ const $<1$. Hence if $\chi$ is contained in a closed ball of radius $R$ in $\tilde{E}_{k}$ with $k \geqq 3$, such that:

$$
R c<1 \text {, }
$$

condition 1 is also satisfied. We conclude that in this case the coefficients $c_{k}^{\prime}$ can be chosen to depend only on $R$ and $T$. We therefore choose a fixed $R$, a priori, satisfying (2.7) and shall regard the $c_{k}^{\prime}$ as depending only on $T$.

\section{The Convergent Iteration Method}

Einstein's field equations for the metric $g$

$$
R^{\mu v}=T^{\mu v}-\frac{1}{2} T g^{\mu v}
$$

do not allow to prescribe $T^{\mu v}$ as a given field on a manifold because (3.1) implies

$$
T_{; v}^{\mu v}=0 \text {. }
$$

If one however imposes the condition of harmonic coordinates (3.1) becomes the reduced Einstein equation

$$
R_{h}^{\mu v}=T^{\mu v}-\frac{1}{2} T g^{\mu v}=\frac{1}{2} \varrho^{\mu \nu} .
$$

This equation has solutions for arbitrary prescribed $\varrho^{\mu \nu}$. The solution is however only a solution of (3.1) if 1) the data for (3.3) satisfy the constraints and 2) if the solution $g^{\mu v}$ and $T^{\mu v}$ defined from $\varrho^{\mu v}$ and $g^{\mu v}$ satisfy $T_{; v}^{\mu v}=0$. 
We are going to consider solutions of (3.3) with given $\varrho$ and trivial Cauchy data, namely:

$$
(g)_{0}=\eta, \quad\left(\partial_{t} g\right)_{0}=0
$$

because we want to study how the source generates the field.

Expressed in terms of $g(3.3)$ becomes

$$
\square_{g} g=f(g, D g)+\varrho
$$

with

$$
f(g, D g)=P_{g}(D g, D g),
$$

where $P_{g}$ is a bilinear form with coefficients which are rational functions of $g^{\mu \nu}$.

In coordinates we have

$$
g^{\alpha \beta} D_{\alpha} D_{\beta} g^{\mu \nu}=P_{\alpha \beta}^{\mu \nu} \stackrel{\rho \sigma}{\rho} D_{\varrho} g^{\alpha \beta} D_{\sigma} g^{j \delta}+Q^{\mu \nu} .
$$

We can write $P_{g}$ in the following form:

$$
P_{g}=I+H_{h},
$$

where $I=P_{\eta}$ is a combination of $\delta_{v}^{\mu}, \eta^{\mu v}$ and $\eta_{\mu v}$ and we have defined $h=g-\eta$. We shall denote $|I|=p$.

Lemma 4. If $h \in \tilde{E}_{s}$ with $s \geqq 2$, and $g=\eta+h$ is non-degenerate, then $H_{h} \in \tilde{E}_{s}$, and if in addition $\|h\|_{s}$ is sufficiently small, there exist constants $q$ and $r$ such that:

i) $\left\|H_{h}\right\|_{l} \leqq q\|h\|_{l}$,

ii) $\left\|H_{h_{1}}-H_{h_{2}}\right\|_{l}<r\left\|h_{1}-h_{2}\right\|_{l}$ for each $l: 0 \leqq l \leqq s$.

Proof. First $H_{h}$ is a rational function of $h$, namely a polynomial in $h$ with denominator a power of det $g$. Since $g$ is non-degenerate, the denominator is different from zero. Therefore $H_{h}$ is an analytic function and corollary 1 implies $H_{h} \in \tilde{E}_{s}$. Then i) and ii) follow from the fact that $H_{h}$ contains no constant term and $\|h\|_{s}$ is sufficiently small.

We associate with the reduced Einstein equations the linear system obtained by replacing $g$ in all terms except the second derivatives by a given tensor field $\gamma$, having trivial Cauchy data. That is :

$$
\square_{\gamma} g=f(\gamma, D \gamma)+\underline{Q} .
$$

Let us set $\chi=\gamma-\eta$.

Lemma 5. If $\chi \in \tilde{E}_{s}$ with $s \geqq 3$ and $\gamma=\eta+\chi$ non-degenerate, then $f(\gamma, D \gamma) \in \tilde{E}_{s-1}$, and if in addition $\|\chi\|_{S}$ is sufficiently small, it holds:

$$
\|f(\gamma, D \gamma)\|_{s-1} \leqq 2 a_{s-1} p\|\chi\|_{s}^{2} \text {. }
$$

Proof. We write $f(\gamma, D \gamma)$ as:

$$
\begin{aligned}
f(\gamma, D \gamma) & =P_{\gamma}(D \gamma, D \gamma) \\
& =I(D \chi, D \chi)+H_{\lambda}(D \chi, D \chi) .
\end{aligned}
$$


As $D \chi \in \tilde{E}_{s-1}$ with $s-1 \geqq 2$, Corollary 1 together with Lemma 4 imply $f \in \tilde{E}_{s-1}$. Further, if $\|\chi\|_{s-1}$ is sufficiently small from Lemma 4 i) we have:

$$
\begin{aligned}
\|f\|_{s-1} & \leqq a_{s-1}\left(p+q a_{s-1}\|\chi\|_{s-1}\right)\|\chi\|_{s}^{2} \\
& \leqq 2 a_{s-1} p\|\chi\|_{s}^{2}
\end{aligned}
$$

provided that

$$
q a_{s-1}\|\|_{s-1} \leqq p
$$

Lemma 6. If $\chi_{1}, \chi_{2}$ are in the closed ball of radius $b_{s}$ in $\tilde{E}_{s}, s \geqq 3$, and $b_{s}$ sufficiently small, the following inequality holds for each $l$ such that $1 \leqq l \leqq s$

$$
\left\|f\left(\chi_{1}, D \chi_{1}\right)-f\left(\chi_{2}, D \chi_{2}\right)\right\|_{l-1} \leqq 4 p a_{s-1, l-1} b_{s}\left\|\chi_{1}-\chi_{2}\right\|_{l} .
$$

Proof. We write

$$
\begin{aligned}
f\left(\chi_{1}, D \chi_{1}\right)-f\left(\chi_{2}, D \chi_{2}\right)= & I\left(D\left(\chi_{1}-\chi_{2}\right), D\left(\chi_{1}+\chi_{2}\right)\right)+H_{\chi_{1}}\left(D\left(\chi_{1}-\chi_{2}\right), D\left(\chi_{1}+\chi_{2}\right)\right) \\
& +\left(H_{\chi_{1}}-H_{\chi_{2}}\right)\left(D \chi_{2}, D \chi_{2}\right) .
\end{aligned}
$$

We shall estimate the $\tilde{E}_{l-1}$-norm of each of the three terms in the above expression separately. For the first term using Lemma $1\left(\tilde{E}_{s-1} \times \tilde{E}_{l-1} \rightarrow \tilde{E}_{l-1}\right)$

$$
\begin{aligned}
& \left\|I\left(D\left(\chi_{1}-\chi_{2}\right), D\left(\chi_{1}+\chi_{2}\right)\right)\right\| \\
& \quad \leqq p a_{s-1 . l-1}\left\|\chi_{1}+\chi_{2}\right\|_{s}\left\|\chi_{1}-\chi_{2}\right\|_{l} \leqq 2 p a_{s-1, l-1} b_{s}\left\|\chi_{1}-\chi_{2}\right\|_{l} .
\end{aligned}
$$

For the second term we use Lemma $1\left(\tilde{E}_{s-1} \times \tilde{E}_{l-1} \rightarrow \tilde{E}_{l-1}\right)$ twice as well as Lemma 4i)

$$
\begin{aligned}
& \left\|H_{\chi_{1}}\left(D\left(\chi_{1}-\chi_{2}\right), D\left(\chi_{1}+\chi_{2}\right)\right)\right\|_{l-1} \\
& \quad \leqq a_{s-1, l-1}\left\|H_{l_{1}}\right\|_{s-1}\left\|D\left(\chi_{1}-\chi_{2}\right) \otimes D\left(\chi_{1}+\chi_{2}\right)\right\|_{l-1} \\
& \quad \leqq q\left(a_{s-1, l-1}\right)^{2}\left\|\chi_{1}\right\|_{s-1}\left\|\chi_{1}+\chi_{2}\right\|_{s}\left\|\chi_{1}-\chi_{2}\right\|_{l} \\
& \quad \leqq 2 q\left(a_{s-1, l-1}\right)^{2} b_{s}^{2}\left\|\chi_{1}-\chi_{2}\right\|_{l} .
\end{aligned}
$$

For the third term we use Lemma $1\left(\tilde{E}_{s-1} \times \tilde{E}_{l-1} \rightarrow \tilde{E}_{l-1}\right)$, Corollary 1 $\left(\tilde{E}_{s-1} \times \tilde{E}_{s-1} \rightarrow \tilde{E}_{s-1}\right)$ and Lemma 4 ii) to get:

$$
\begin{aligned}
& \left\|\left(H_{\chi_{1}}-H_{\chi_{2}}\right)\left(D \chi_{2}, D \chi_{2}\right)\right\|_{l-1} \\
& \quad \leqq a_{s-1, l-1}\left\|H_{\chi_{1}}-H_{\chi_{2}}\right\|_{l-1}\left\|D \chi_{2} \otimes D \chi_{2}\right\|_{s-1} \\
& \quad \leqq r a_{s-1} a_{s-1, l-1}\left\|\chi_{2}\right\|_{s}^{2}\left\|\chi_{1}-\chi_{2}\right\|_{l-1} \\
& \quad \leqq r a_{s-1} a_{s-1, l-1} b_{s}^{2}\left\|\chi_{1}-\chi_{2}\right\|_{l}
\end{aligned}
$$

Combining (3.12)-(3.14) we obtain

$$
\begin{aligned}
& \left\|f\left(\chi_{1}, D \chi_{1}\right)-f\left(\chi_{2}, D \chi_{2}\right)\right\|_{l-1} \\
& \quad \leqq a_{s-1, l-1} b_{s}\left[2 p+\left(2 q a_{s-1, l-1}+r a_{s-1}\right) b_{s}\right]\left\|\chi_{1}-\chi_{2}\right\|_{l},
\end{aligned}
$$

from which we recover the lemma if:

$$
\left(2 q a_{s-1, l-1}+r a_{s-1}\right) b_{s} \leqq 2 p .
$$


Theorem 1 and Lemma 5 imply now that if $\varrho \in \tilde{E}_{k-1}$ and $\chi \in \tilde{E}_{k}$ with $k \geqq 3$ and if in addition $\|\chi\|_{\tilde{E}_{k}}$ is sufficiently small, then Eq. (3.9) has a solution $g=\eta+h$ for trivial Cauchy data such that $h \in \tilde{E}_{k}$. Furthermore, the solution is unique in $\tilde{E}_{1}$. Hence we can define a map $\Phi: \chi \mapsto h$ from a sufficiently small ball in $\tilde{E}_{k}$ into $\tilde{E}_{k}$, for $k \geqq 3$. We shall now prove:

Lemma 7. If $\varrho \in \tilde{E}_{k-1}, k \geqq 3$ and $b_{k}$ is sufficiently small, $\Phi$ maps the closed ball of radius $b_{k}$ in $\tilde{E}_{k}$ into the closed ball of radius $b_{k}^{\prime}$ in the same space, where:

$$
b_{k}^{\prime}=c_{k}^{\prime}\left(2 p a_{k-1} b_{k}^{2}+\|Q\|_{k-1}\right) \text {. }
$$

Proof. From Theorem 1 we have:

$$
\|h\|_{k} \leqq c_{k}^{\prime}\left(\|f\|_{k-1}+\|\varrho\|_{k-1}\right),
$$

which, taking into account Lemma 5 gives:

$$
\|h\|_{k} \leqq c_{k}^{\prime}\left(2 p a_{k-1}\|\chi\|_{k}^{2}+\|Q\|_{k-1}\right),
$$

provided that (3.10) is satisfied. The above inequality implies the lemma.

Corollary 2. If $\varrho \in \tilde{E}_{k-1}, k \geqq 3$ and $\|\varrho\|_{k-1}$ is sufficiently small, $\Phi$ maps the closed ball of radius $B_{k}$ in $\tilde{E}_{k}$ into itself, where

$$
B_{k}=\frac{1-\sqrt{1-8\left(c_{k}^{\prime}\right)^{2} a_{k-1} p\|\varrho\|_{k-1}}}{4 c_{k}^{\prime} a_{k-1} p} .
$$

Proof. From Lemma $7, b_{k}^{\prime} \leqq b_{k}$ if

$$
c_{k}^{\prime}\left(2 p a_{k-1} b_{k}^{2}+\|\varrho\|_{k-1}\right) \leqq b_{k} .
$$

The above inequality can be satisfied provided that

$$
8\left(c_{k}^{\prime}\right)^{2} a_{k-1} p\|\varrho\|_{k-1} \leqq 1 .
$$

Then the minimal $b_{k}$ satisfying (3.19) is the smallest of the two roots of the corresponding equality, which is (3.18). Furthermore,

$$
B_{k} \leqq 2 c_{k}^{\prime}\|\varrho\|_{k-1} .
$$

Consequently, (3.10) is satisfied provided that

$$
2 q c_{k}^{\prime} a_{k-1}\|\varrho\|_{k-1} \leqq p
$$

Using the map $\Phi$ we define the sequence $\left\{h_{n}\right\}$ by:

$$
\begin{aligned}
h_{0} & =0 \\
h_{n+1} & =\Phi\left(h_{n}\right) .
\end{aligned}
$$

Corollary 3. If $\varrho \in \tilde{E}_{k-1}, k \geqq 3$ and $\|\varrho\|_{k-1}$ is sufficiently small, the sequence $\left\{h_{n}\right\}$ is contained in the closed ball of radius $B_{k}$ in $\tilde{E}_{k}$.

The sequence $\left\{h_{n}\right\}$ is shown to be a Cauchy sequence by demonstrating that $\Phi$ is a contracting map. 
Lemma 8. If $\chi_{1}, \chi_{2}$ are in a closed ball of radius $b$ in $\tilde{E}_{3}, b$ is sufficiently small and $\varrho \in \tilde{E}_{2}$, their images under $\Phi$ satisfy the following inequality:

$$
\left\|\Phi\left(\chi_{1}\right)-\Phi\left(\chi_{2}\right)\right\|_{1} \leqq \lambda\left(b, b^{\prime}\right)\left\|\chi_{1}-\chi_{2}\right\|_{1},
$$

where:

$$
\lambda\left(b, b^{\prime}\right)=c_{1}^{\prime}\left(a_{1,1,0} b^{\prime}+4 p a_{2,0} b\right)
$$

and $b^{\prime}$ is given in terms of $b$ and $\|\varrho\|_{2}$ by (3.17) for $k=3$.

Proof. Let $h_{1}=\Phi\left(\chi_{1}\right)$ and $h_{2}=\Phi\left(\chi_{2}\right)$. Subtracting (3.9) for $h_{2}$ from the same equation for $h_{1}$ we obtain: $\left(\gamma_{1}=\eta+\chi_{1}, \gamma_{2}=\eta+\chi_{2}\right)$

$$
\square_{\gamma_{1}} h_{1}-\square_{\gamma_{2}} h_{2}=f\left(\gamma_{1}, D \gamma_{1}\right)-f\left(\gamma_{2}, D \gamma_{2}\right)
$$

or equivalently,

$$
\square_{\gamma_{1}}\left(h_{1}-h_{2}\right)=-\left(\square_{\gamma_{1}}-\square_{\gamma_{2}}\right) h_{2}+f\left(\gamma_{1}, D \gamma_{1}\right)-f\left(\gamma_{2}, D \gamma_{2}\right)
$$

Applying Theorem 1 for $k=1$ to (3.26), where we consider $h_{1}-h_{2}$ as the unknown $\phi$ and the right-hand side as the given source $\varrho$, we obtain

$$
\left\|h_{1}-h_{2}\right\|_{1} \leqq c_{1}^{\prime}\left\{\left\|\left(\chi_{1}-\chi_{2}\right) \cdot D^{2} h_{2}\right\|_{0}+\left\|f\left(\chi_{1}, D \chi_{1}\right)-f\left(\chi_{2}, D \chi_{2}\right)\right\|_{0}\right\} .
$$

Using Lemma $1\left(\tilde{E}_{1} \times \tilde{E}_{1} \rightarrow \tilde{E}_{0}\right)$ we estimate the first term on the right by:

$$
\begin{aligned}
\left\|\left(\chi_{1}-\chi_{2}\right) \cdot D^{2} h_{2}\right\|_{0} & \leqq a_{1,1,0}\left\|\chi_{1}-\chi_{2}\right\|_{1}\left\|h_{2}\right\|_{3} \\
& \leqq a_{1,1,0} b^{\prime}\left\|\chi_{1}-\chi_{2}\right\|_{1},
\end{aligned}
$$

where we have applied Lemma 7 with $k=3$. To estimate the second term on the right in (3.27) we use Lemma 6 with $s=3, l=1$ :

$$
\left\|f\left(\chi_{1}, D \chi_{1}\right)-f\left(\chi_{2}, D \chi_{2}\right)\right\|_{0} \leqq 4 p a_{2,0} b\left\|\chi_{1}-\chi_{2}\right\|_{1} .
$$

Substituting (3.28) and (3.29) in (3.27) we recover the lemma.

Corollary 4. If $\varrho \in \tilde{E}_{2}$ and $\|\varrho\|_{2}$ is sufficiently small, the map $\Phi$ is contractive in the $\tilde{E}_{1}$ norm on the closed ball of radius $B$ in $\tilde{E}_{3}$.

Proof. Under the hypotheses of the corollary, Corollary 2 ensures us that the closed ball of radius $B$ [given by (3.18) with $k=3$ ] in $\tilde{E}_{3}$ is mapped into itself by $\Phi$. The rest follows from Lemma 8 provided that:

$$
\Lambda=\lambda(B, B)=c_{1}^{\prime}\left(a_{1,1,0}+4 p a_{2,0}\right) B<1
$$

which, in view of (3.21) holds if

$$
2 c_{1}^{\prime} c_{3}^{\prime}\left(a_{1,1,0}+4 p a_{2,0}\right)\|\varrho\|_{2}<1 \text {. }
$$

The contractiveness of $\Phi$ allows us to prove an existence and uniquess theorem.

Theorem 2. If $\varrho \in \tilde{E}_{k-1}$ with $k \geqq 3$ and if $\|\varrho\|_{\tilde{E}_{k-1}}$ is sufficiently small, the reduced Einstein equations with source $\varrho$ have one and only one solution $g$ on $I \times \mathbb{R}^{3}$ with trivial Cauchy data, where $h=g-\eta$ is in $\tilde{E}_{k}$ and $g$ is regularly hyperbolic. 
Proof. By Lemma 8, if the hypotheses of the theorem are satisfied, the map $\Phi$ is contractive in the $\tilde{E}_{1}$-norm on the closed ball of radius $B$ in $\tilde{E}_{3}$. By the contraction mapping principle, $\Phi$ has a unique fixed point $h$ in $\tilde{E}_{1}$. To show that $h$ is also in $\tilde{E}_{k}$ we argue as follows: By Corollary 3, for each multi-index $\alpha$ with $0 \leqq|\alpha| \leqq k$, the sequence $\left(D^{x} h_{n}\right)_{t}$ is uniformly (in $n$ and $t$ ) bounded in the $L^{2}\left(\mathbb{R}^{3}\right)$-norm. Thus we can extract a subsequence which converges weakly to some $\left(h^{\alpha}\right)_{t} \in L^{2}\left(\mathbb{R}^{3}\right)$. It is easily seen that $h^{\alpha}=D^{\alpha} h^{0}$. Since the weak limit of a measurable map is measurable, we can show that $t \mapsto\left\|h^{0}\right\|_{k}^{s_{t}}$ is measurable, hence $h^{0} \in \tilde{E}_{k}$. Finally, it is evident that $h^{0}=h$ apart from subsets of zero measure in each $\Sigma_{t}[9-11]$.

\section{The Asymptotic Iteration Method}

The convergent iteration method, which was analyzed in the previous sections, cannot be constructed in practice, since it requires the knowledge of the Green's function of a given metric for the Cauchy problem posed. This is the reason why the following iteration is used instead in actual calculations, which uses only the Green's function of the Minkowski metric, i.e., the truncated retarded integral. This is essentially the so-called "fast motion iteration method" $[5,12,13]$. This method is defined by associating with the reduced Einstein equations (3.5) the following linear system

$$
\square \bar{g}=f(\gamma, D \gamma)+Q-(\square,-\square) \gamma,
$$

where $\gamma$ is, as in Eq. (3.9), a given tensor field and $\square \equiv \square_{\eta}$.

Let $\varrho \in \tilde{E}_{k-2}$ and $\chi \in \tilde{E}_{k}, k \geqq 3$ such that $\gamma=\eta+\chi$ is non-degenerate. Then we know from Lemma 5 that $f(\gamma, D \gamma) \in \tilde{E}_{k-1}$. Further, the last term on the right which is $\chi \cdot D^{2} \chi \in \tilde{E}_{k-2}$. Hence the effective source in (4.1) is in $\tilde{E}_{k-2}$ and Theorem 1 (with $\gamma=\eta$ ) gives us a solution $\bar{h}=\bar{g}-\eta \in \tilde{E}_{k-1}$ having trivial Cauchy data, unique in $\tilde{E}_{1}$. Thus we can define a map $\Psi: \chi \mapsto \bar{h}$ which sends a sufficiently small ball in $\tilde{E}_{k}$ into $\tilde{E}_{k-1}$. This map is thus not, as $\Phi$ is, a map from one space into itself. The reason for this is the appearance of second derivatives on the right hand side of (4.1) which causes the loss of one order of differentiability.

Using the map $\Psi$ we define the sequence $\left\{\bar{h}_{n}\right\}$ by

$$
\begin{aligned}
\bar{h}_{0} & =0 \\
\bar{h}_{n+1} & =\Psi\left(\bar{h}_{n}\right) .
\end{aligned}
$$

Lemma 9. The map $\Psi$ sends the ball of radius $b_{k}$ in $\tilde{E}_{k}, k \geqq 3$, into the ball of radius $b_{k-1}^{\prime \prime}$ in $\tilde{E}_{k-1}$, where:

$$
b_{k-1}^{\prime \prime}=c_{k-1}\left\{\|\varrho\|_{k-2}+\left(2 a_{k-1} p+a_{k-1 . k-2}\right) b_{k}^{2}\right\} .
$$

The coefficients $c_{k}$ are the coefficient $c_{k}^{\prime}$ of Theorem 1 in the special case $\gamma=\eta$.

Proof. Applying Theorem 1 with $\gamma=\eta$ gives us

$$
\|\bar{h}\|_{E_{k-1}} \leqq c_{k-1}\left\{\|Q\|_{k-2}+\|f(\chi, D \chi)\|_{k-2}+\left\|\chi \cdot D^{2} \chi\right\|_{k-2}\right\} .
$$

Using Lemma $1\left(\tilde{E}_{k-1} \times \tilde{E}_{k-2} \rightarrow \tilde{E}_{k-2}\right), k \geqq 3$

$$
\left\|\chi \cdot D^{2} \chi\right\|_{k-2} \leqq a_{k-1, k-2}\|\chi\|_{k-1}\|\chi\|_{k} \leqq a_{k-1, k-2}\|\chi\|_{k}^{2} .
$$


The term in $f$ is estimated using Lemma 5 .

$$
\|f(\chi, D \chi)\|_{k-2} \leqq\|f(\chi, D \chi)\|_{k-1} \leqq 2 a_{k-1} p\|\chi\|_{k}^{2} .
$$

Substituting (4.5), (4.4) into (4.3) we get Lemma 9.

Corollary 5. If $\varrho \in \tilde{E}_{n+k-3}, k \geqq 3$ and $\|\varrho\|_{n+k-3}$ is sufficiently small then $\bar{h}_{n} \in \tilde{E}_{k-1}$ and

$$
\left\|\bar{h}_{n}\right\|_{k-1} \leqq 2 c_{k-1}\|Q\|_{n+k-3} .
$$

The proof is by induction on $n$ using Lemma 9.

The analog for $\Psi$ of the contraction property of $\Phi$ is :

Lemma 10. If $\chi_{1}, \chi_{2}$ are in a sufficiently small ball of radius $b_{k}$ in $\tilde{E}_{k}, k \geqq 3$ their images $\bar{h}_{1}, \bar{h}_{2}$ under $\Psi$ satisfy:

$$
\left\|\bar{h}_{1}-\bar{h}_{2}\right\|_{k-1} \leqq \mu_{k}\left\|\chi_{1}-\chi_{2}\right\|_{k}
$$

where: $\mu_{k}=2 c_{k-1}\left(2 p a_{k-1}+a_{k-1, k-2}\right) b_{k}$.

Proof. $\bar{h}_{1}, \bar{h}_{2}$ satisfy the Eq. (4.1), hence their difference satisfies

$$
\square\left(\bar{h}_{1}-\bar{h}_{2}\right)=f\left(\chi_{1}, D \chi_{1}\right)-f\left(\chi_{2}, D \chi_{2}\right)-\left(\chi_{1} \cdot D^{2} \chi_{1}-\chi_{2} \cdot D^{2} \chi_{2}\right) .
$$

We write the second term as

$$
\chi_{1} \cdot D \chi_{1}-\chi_{2} \cdot D^{2} \chi_{2}=\left(\chi_{1}-\chi_{2}\right) \cdot D^{2} \chi_{1}+\chi_{2} \cdot D^{2}\left(\chi_{1}-\chi_{2}\right) .
$$

Using Lemma $1\left(\tilde{E}_{k-1} \times \tilde{E}_{k-2} \rightarrow \tilde{E}_{k-2}\right), k \geqq 3$ we get

$$
\begin{aligned}
& \left\|\left(\chi_{1}-\chi_{2}\right) \cdot D^{2} \chi_{1}\right\|_{k-2} \leqq a_{k-1, k-2}\left\|\chi_{1}-\chi_{2}\right\|_{k-1}\left\|\chi_{1}\right\|_{k} \\
& \left\|\chi_{2} \cdot D^{2}\left(\chi_{1}-\chi_{2}\right)\right\|_{k-2} \leqq a_{k-1, k-2}\left\|\chi_{2}\right\|_{k-1}\left\|\chi_{1}-\chi_{2}\right\|_{k} .
\end{aligned}
$$

Hence combining both gives

$$
\left\|\chi_{1} \cdot D^{2} \chi_{1}-\chi_{2} \cdot D^{2} \chi_{2}\right\|_{k-2} \leqq 2 a_{k-1, k-2} b_{k}\left\|\chi_{1}-\chi_{2}\right\|_{k} .
$$

The terms in $f$ are estimated using Lemma 6 for $l=s=k$ :

$$
\begin{aligned}
\left\|f\left(\chi_{1}, D \chi_{1}\right)-f\left(\chi_{2}, D \chi_{2}\right)\right\|_{k-2} & \leqq\left\|f\left(\chi_{1}, D \chi_{1}\right)-f\left(\chi_{2}, D \chi_{2}\right)\right\|_{k-1} \\
& \leqq 4 p a_{k-1} b_{k}\left\|\chi_{1}-\chi_{2}\right\|_{k} .
\end{aligned}
$$

Applying Theorem 1 to (4.6) and using estimates (4.7) and (4.8) for the effective source we obtain Lemma 10.

Corollary 6. If $\varrho \in \tilde{E}_{n+k-3}, n \geqq 1, k \geqq 3$ and $\|\varrho\|_{n+k-3}$ sufficiently small then

$$
\left\|\bar{h}_{n}-\bar{h}_{n-1}\right\|_{k-1} \leqq e_{n, k-1}\|\varrho\|_{n+k-3}^{n},
$$

where the coefficients $e_{n, k}$ are given by the recursion relation

$$
e_{n+1, k-1}=4 c_{k-1} c_{k}\left(2 p a_{k-1}+a_{k-1, k-2}\right) e_{n, k}
$$


with

$$
e_{1, k}=c_{k} .
$$

The proof is by induction on $n$ using Lemma 10 .

Lemmas 9 and 10 show that there is a loss of 1 order of differentiability at each step of the iteration method of this section. Therefore the sequence $\left\{\bar{h}_{n}\right\}$ can not be expected to converge in general, even if $\varrho$ is in $\tilde{E}_{k}$ for all $k$. It will however be shown in Sect. 6 that it is asymptotic to the exact solution.

\section{Comparison Between the Convergent and Asymptotic Iteration}

In the previous sections we discussed the convergent and asymptotic iteration methods separately. In this section we shall fulfill the aim of this paper by establishing relations between the two iteration methods.

Lemma 11. Let $\chi$ be in a ball of radius $b$ in $\tilde{E}_{3}$, with $b$ sufficiently small. Then

$$
\|\Phi(\chi)-\Psi(\chi)\|_{1} \leqq v b\|\chi-\Psi(\chi)\|_{2},
$$

where $v=c_{1}^{\prime} a_{2,0}$.

Proof. Writing $\Phi(\chi)=h, \Psi(\chi)=\bar{h}$ the following equations hold [(3.9), (4.1)]

$$
\begin{aligned}
& \square_{\gamma} h=f(\chi, D \chi)+\varrho, \\
& \square \tilde{h}=f(\chi, D \chi)+\varrho-\chi \cdot D^{2} \chi,
\end{aligned}
$$

where $\gamma=\eta+\chi$.

Subtracting (5.2) from (5.1) and adding on both sides of the resulting equation the quantity $\left(\square-\square_{\gamma}\right) \bar{h}=-\chi \cdot D^{2} h$ one gets

$$
\square_{\gamma}(h-\bar{h})=\chi \cdot D^{2}(\chi-\bar{h}) .
$$

Theorem 1 implies

$$
\begin{aligned}
\|h-\bar{h}\|_{1} & \leqq c_{1}^{\prime}\left\|\chi \cdot D^{2}(\chi-\bar{h})\right\|_{0} \\
& \leqq c_{1}^{\prime} a_{2.0}\|\chi\|_{2}\|\chi-\bar{h}\|_{2},
\end{aligned}
$$

from which we recover the lemma.

We shall use the above lemma in

Lemma 12. Let $\chi_{1}, \chi_{2}$ be in a ball of radius $b$ in $\tilde{E}_{3}$ with $b$ sufficiently small and let $Q$ be in $\tilde{E}_{2}$. Then

$$
\left\|\Phi\left(\chi_{1}\right)-\Psi\left(\chi_{2}\right)\right\|_{1} \leqq \lambda\left(b, b^{\prime}\right)\left\|\chi_{1}-\chi_{2}\right\|_{1}+v b\left\|\chi_{2}-\Psi\left(\chi_{2}\right)\right\|_{2},
$$

where $\lambda\left(b, b^{\prime}\right)$ is defined in (3.24).

Proof. We have

$$
\left\|\Phi\left(\chi_{1}\right)-\Psi\left(\chi_{2}\right)\right\|_{1} \leqq\left\|\Phi\left(\chi_{1}\right)-\Phi\left(\chi_{2}\right)\right\|_{1}+\left\|\Phi\left(\chi_{2}\right)-\Psi\left(\chi_{2}\right)\right\|_{1} .
$$

Estimating the first term on the right using Lemma 8 and the second using Lemma 11, we recover the lemma. 
Theorem 3. Let $\varrho$ be in $\tilde{E}_{m}, m \geqq 2$ and $\|\varrho\|_{m}$ sufficiently small. Then the difference between the iterative $\bar{h}_{m}$ and the exact solution $h$ satisfies the following estimate:

$$
\left\|h-\bar{h}_{m}\right\|_{1} \leqq j_{m}\|\varrho\|_{m}^{m+1}
$$

where the $j_{m}$ 's are constants which depend only on the size $T$ of the interval $I$.

Proof. We substitute in Lemma 12 for $\chi_{1}, \chi_{2}$ the iterates $h_{n}$ and $\bar{h}_{n}$ respectively, and hence for $\Phi\left(\chi_{1}\right), \Psi\left(\chi_{2}\right)$ their successors $h_{n+1}, \bar{h}_{n+1}$ in the corresponding sequences. The assumptions of the lemma require $h_{n}$ and $\bar{h}_{n}$ to be contained in a ball of radius $b$ in $\tilde{E}_{3}$. From Corollary 3 , if $\varrho \in \tilde{E}_{2}$ and $\|\varrho\|_{2}$ is sufficiently small, we have:

$$
\left\|h_{n}\right\|_{3} \leqq 2 c_{3}^{\prime}\|\varrho\|_{2}
$$

[taking into account (3.21)]. On the other hand if $\varrho \in \tilde{E}_{n+1}, n \geqq 1$ with $\|\varrho\|_{n+1}$ sufficiently small, Corollary 5 gives us:

$$
\left\|\bar{h}_{n}\right\|_{3} \leqq 2 c_{3}\|Q\|_{n+1} .
$$

From (5.4) and (5.5) we conclude that both $h_{n}$ and $\bar{h}_{n}$ are contained in the ball of radius

$$
b=2 c_{3}^{\prime}\|\varrho\|_{n+1}
$$

in $\tilde{E}_{3}$. Then (3.17) gives

$$
b^{\prime}=c_{3}^{\prime}\left(2 p a_{2} b^{2}+\|\varrho\|_{2}\right) \leqq 2 c_{3}^{\prime}\|\varrho\|_{n+1}=b,
$$

where the last step holds if

$$
8 p a_{2}\left(c_{3}^{\prime}\right)^{2}\|\varrho\|_{n+1} \leqq 1 .
$$

Substituting (5.6) and (5.7) in (3.24) we obtain

$$
\lambda\left(b, b^{\prime}\right) \leqq \alpha\|\varrho\|_{n+1},
$$

where:

$$
\alpha=2 c_{1}^{\prime} c_{3}^{\prime}\left(a_{1,1,0}+4 p a_{2,0}\right) .
$$

Further

$$
v b=\beta\|\varrho\|_{n+1},
$$

where:

$$
\beta=2 c_{1}^{\prime} c_{3}^{\prime} a_{2,0} .
$$

On the other hand Corollary 6 implies

$$
\left\|\chi_{2}-\Psi\left(\chi_{2}\right)\right\|_{2}=\left\|\bar{h}_{n}-\bar{h}_{n+1}\right\|_{2} \leqq e_{n+1,2}\|\varrho\|_{n+1}^{n+1} \text {. }
$$

With the above substitutions Lemma 12 becomes the recursion relation

$$
\left\|h_{n+1}-\bar{h}_{n+1}\right\|_{1} \leqq \alpha\|\varrho\|_{n+1}\left\|h_{n}-\bar{h}_{n}\right\|_{1}+e_{n+1,2}\|\varrho\|_{n+1}^{n+2} \text {. }
$$


Taking into account the fact that $h_{1}=\bar{h}_{1}$, if $\varrho \in \tilde{E}_{m}, m \geqq 2$ and $\|\varrho\|_{m}$ sufficiently small, using induction on $n$ for $1 \leqq n \leqq m-1,(5.13)$ leads to:

$$
\left\|h_{m}-\bar{h}_{m}\right\|_{1} \leqq f_{m}\|\varrho\|_{m}^{m+1}
$$

where:

$$
f_{m}=\sum_{i=2}^{m} \beta e_{i, 2} \alpha^{m-i} .
$$

The difference between the exact solution $h$ and the iterate $h_{n}$ of the convergent sequence satisfies the estimate:

$$
\left\|h-h_{n}\right\|_{1} \leqq \frac{\Lambda^{n}}{1-\Lambda}\left\|h_{1}-h_{0}\right\|_{1}=\frac{\Lambda^{n}}{1-\Lambda}\left\|h_{1}\right\|_{1},
$$

where $\Lambda$ is the contraction constant, $\Lambda<1$, given by (3.30):

$$
\Lambda \leqq \alpha\|Q\|_{2} \text {. }
$$

The estimate (5.16) follows from:

$$
\left\|h_{n+1}-h_{n}\right\|_{1} \leqq \Lambda\left\|h_{n}-h_{n-1}\right\|_{1}
$$

(contractiveness of $\Phi$ ). If then

$$
2 \alpha\|\varrho\|_{2} \leqq 1,
$$

we get from $(5.16):\left(\right.$ since $\left.\left\|h_{1}\right\|_{1} \leqq \mathcal{C}_{1}\|\varrho\|_{0}\right)$

$$
\left\|h-h_{n}\right\|_{1} \leqq 2 c_{1} \alpha^{n}\|Q\|_{2}^{n+1} .
$$

Combining finally (5.14) with (5.18) we obtain:

$$
\left\|h-\bar{h}_{m}\right\|_{1} \leqq j_{m}\|Q\|_{m}^{m+1},
$$

where:

$$
j_{m}=f_{m}+2 c_{1} \alpha^{m} .
$$

\section{Conclusions}

Theorem 3 of the previous section shows that the sequence $\left\{\bar{h}_{n}\right\}$ calculated by the "fast motion" iteration method which uses only the Minkowski Green's function is asymptotic to the exact solution $h$. That is, for fixed $n$, if $Q \rightarrow 0$ in the $\tilde{E}_{n}$ norm then $\bar{h}_{n} \rightarrow h$ in the $\tilde{E}_{1}$ norm as $\|Q\|_{n}^{n+1}$. Furthermore we deduced precise error estimates for the sequence $\left\{\bar{h}_{n}\right\}$ in $\tilde{E}_{1}$ norm. In applying the estimates given to physical cases, a choice of length scale should beforehand be made, since neither the constants $c_{k}$, $c_{k}^{\prime}$ nor the $\tilde{E}_{k}$ norms are scale invariant. Perhaps the most convenient such choice is to take the time interval $T=1$.

On the other hand domain of dependence considerations indicate that the sequence $\left\{\bar{h}_{n}\right\}$ does not converge to $h$, except in the case where $\varrho$ is analytic.

Several generalizations of our results are possible using essentially the same methods: First, we can admit general Cauchy data instead of trivial. Second, we 
can give error estimates in the $\tilde{E}_{l}$ norm, with $l \geqq 1$. Third, the treatment applies to any weakly coupled quasilinear hyperbolic system in any dimension, which has the same structure as the reduced Einstein equations.

Acknowledgements. The following work was stimulated by lectures of J. Ehlers who pointed out repeatedly the need for justifying formal approximation methods in $G R$ and who suggested the posssibility to make rigorous certain "plausible" error estimates sketched in [5] and [6].

\section{References}

1. Choquet-Bruhat, Y.: Acta Math. 88, 141 (1952)

2. Hawking, S., Ellis, G.: The large scale structure of space-time. Cambridge: Cambridge University Press 1973

3. Fischer, A., Marsden, J. : Commun. Math. Phys. 28, 1-38 (1972)

4. Müller zum Hagen, H., Seifert, H.: Gen. Rel. Grav. 8, 259 (1977)

5. Synge, J.: Proc. Roy. Irish Acad. A 69, 11 (1970)

6. Ehlers, J.: Proc. Intern. School of Relativistic Astrophysics (3rd Course). Erıce/Sicila, preprint MPI-PAE/Astro 138 (1977)

7. Palais, R.: Foundation of global non-linear analysis. New York: Benjamin 1968

8. Adams, R. : Sobolev spaces. New York: Academic Press 1975

9. Choquet-Bruhat, Y., Christodoulou, D., Francaviglia, M.: Ann. Inst. Henri Poincaré 29, 241 (1968)

10. Dionne, P.: J. Math. Jérusalem 10, 1 (1962)

11. Bourbaki, J.N.: XIII. Foctions d'une variable réelle. Ch. 4, Sect. 5. Paris: Hermann 1966

12. Fock. V.: The theory of space, time, and gravitation. New York. London: Pergamon Press 1964

13. Havas, P., Goldberg, J. : Phys. Rev. 128, 398 (1962)

Communicated by R. Geroch

Received March 22, 1979 
